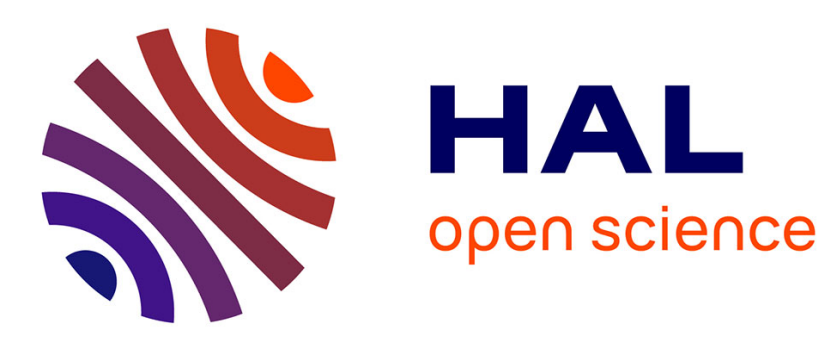

\title{
Étude d'un échangeur à contre-courant gaz-gouttes de chlorure de sodium en cours de cristallisation
}

\author{
M. Desaulty
}

\section{To cite this version:}

M. Desaulty. Étude d'un échangeur à contre-courant gaz-gouttes de chlorure de sodium en cours de cristallisation. Revue de Physique Appliquée, 1980, 15 (2), pp.189-199. 10.1051/rphysap:01980001502018900 . jpa-00244714

\section{HAL Id: jpa-00244714 https://hal.science/jpa-00244714}

Submitted on 1 Jan 1980

HAL is a multi-disciplinary open access archive for the deposit and dissemination of scientific research documents, whether they are published or not. The documents may come from teaching and research institutions in France or abroad, or from public or private research centers.
L'archive ouverte pluridisciplinaire HAL, est destinée au dépôt et à la diffusion de documents scientifiques de niveau recherche, publiés ou non, émanant des établissements d'enseignement et de recherche français ou étrangers, des laboratoires publics ou privés. 


\title{
Étude d'un échangeur à contre-courant gaz-gouttes de chlorure de sodium en cours de cristallisation
}

\author{
M. Desaulty (*) \\ Groupe de Recherches Thermiques du C.N.R.S., associé à l'Ecole Centrale des Arts et Manufactures, Grande Voie des Vignes, \\ 92290 Châtenay Malabry, France
}

(Reçu le 27 juillet 1979, révisé le 7 novembre 1979, accepté le 9 novembre 1979)

\begin{abstract}
Résumé. - Un modèle numérique d'échangeur contre-courant entre un gaz et un sel fondu est présenté. L'énergie du gaz est directement convertie en énergie électrique ou mécanique dans une turbine à gaz. Ceci nécessite que la température de sortie du gaz soit de l'ordre de 750 à $850^{\circ} \mathrm{C}$. Ce résultat peut être obtenu, malgré la mauvaise conductivité du $\mathrm{NaCl}$ et le coefficient de transfert thermique médiocre du gaz, par pulvérisation du sel, ce qui fournit une surface de contact gaz-gouttes très importante.

Un algorithme permettant de déterminer la hauteur nécessaire au refroidissement des gouttes est présenté, ainsi que la répartition de température, vitesses... dans l'échangeur.
\end{abstract}

\begin{abstract}
A numerical model of a counterflow heat exchanger between a gas and a molten salt is presented. The energy of the gas is directly converted into electrical or mechanical energy through a gas-turbine process. This operation requires the energy to be removable at temperature between 750 and $850^{\circ} \mathrm{C}$. The energy can be withdrawn in spite of the bad conductivity of $\mathrm{NaCl}$ and the moderate heat transfer coefficient of the gas, by pulverization of the salt, which gives a very important surface available for heat transfer. Algorithms for estimating the heigh of a vertical column for cooling droplets are described and the distribution of temperatures, velocities... in the exchanger are determined.
\end{abstract}

\section{Nomenclature}

$A$

tension superficielle.

$B i \quad$ nombre de Biot $=\frac{h R_{1}}{\lambda_{\mathrm{s}}}$.

$C_{\mathrm{pg}} \quad$ chaleur massique du gaz.

$C_{\mathrm{pl}} \quad$ chaleur massique du sel liquide.

$C_{\mathrm{ps}} \quad$ chaleur massique du sel solide.

$C_{\mathrm{v}} \quad$ concentration volumique.

$C_{\mathrm{x}} \quad$ coefficient de traînée.

$\mathrm{d} t \quad$ pas de temps.

$g$ accélération de la pesanteur.

$h$ coefficient de convection.

$L \quad$ chaleur latente de changement d'état.

$\stackrel{\circ}{m}_{\mathrm{g}} \quad$ débit masse de gaz.

$\stackrel{\circ}{m}$ débit masse de sel.

$N$ nombre de gouttes émises par seconde.

$\mathrm{Nu}$ nombre de Nusselt.

$P \quad$ pression.

Pr nombre de Prandtl.

$\left(^{*}\right)$ Chercheur au Groupe de Recherches Thermiques du C.N.R.S., associé à l'Ecole Centrale des Arts et Manufactures, 92290 Châtenay-Malabry.

Ces recherches sont conduites avec l'aide de la D.G.R.S.T.
$Q \quad$ quantité de chaleur déstockée depuis l'instant $t=0$ par une goutte.

$Q=\int_{0}^{t} \varphi \mathrm{d} t$.

$R_{1} \quad$ rayon des gouttes.

$R e$ nombre de Reynolds.

$R_{\mathrm{F}} \quad$ rayon de solidification.

$R_{\mathrm{c}} \quad$ rayon de l'échangeur.

$r=\frac{R}{R_{1}}$.

$S_{\mathrm{e}} \quad$ section de l'échangeur.

Ste nombre de Stephan $C_{\mathrm{ps}}\left(T_{\mathrm{f}}-T_{\mathrm{g}}\right) / L$.

$T_{\mathrm{L}} \quad$ température de la phase liquide.

$T_{\mathrm{Le}} \quad$ température de la phase liquide à l'injection.

$T_{\mathrm{S}} \quad$ température de la phase solide.

$T_{\mathrm{F}} \quad$ température de fusion.

$T_{\mathrm{p}} \quad$ température de surface.

$T_{\mathrm{g}} \quad$ température du gaz.

$T_{\text {ge }} \quad$ température d'entrée du gaz.

$T_{\mathrm{gs}} \quad$ température de sortie du gaz.

$T^{+} \quad$ température adimensionnée : $T^{+}=\frac{T-T_{\mathrm{F}}}{\left(T_{\mathrm{f}}-T_{\mathrm{g}}\right)_{\mathrm{e}}}$.

$t \quad$ temps.

$U_{\mathrm{R}} \quad$ vitesse relative $=V_{\mathrm{S}}+V_{\mathrm{g}}$. 
$V_{\mathrm{g}} \quad$ vitesse du gaz.

$V_{\mathrm{s}} \quad$ vitesse des gouttes.

$V_{\mathbf{s i}} \quad$ vitesse initiale des gouttes.

We nombre de Weber.

$x \quad$ abscisse comptée positivement à partir de la section d'entrée des gouttes.

$\rho_{\mathrm{g}} \quad$ masse volumique du gaz.

$\rho_{\mathrm{s}} \quad$ masse volumique du $\mathrm{NaCl}$.

$\lambda_{\mathrm{s}} \quad$ conductivité thermique de la phase solide.

$\lambda_{\mathrm{L}} \quad$ conductivité thermique de la phase liquide.

$\varphi \quad$ flux échangé entre une goutte et le gaz.

$\frac{\stackrel{\circ}{m_{s}}}{\stackrel{\circ}{m}_{\mathrm{g}}}=\eta$ rapport du débit de sel sur le débit de gaz.

$\mu \quad$ viscosité dynamique.

$\tau \quad$ nombre de Fourier ou temps adimensionné.

1. Introduction. - On présente ici une modélisation d'un échangeur direct à contre-courant gazgouttes de chlorure de sodium en cours de solidification : le but est la réalisation d'une unité de stockagedéstockage intégrable dans un cycle de conversion d'énergie à haut niveau thermique. Une des principales difficultés rencontrées sur le plan général au niveau du déstockage est la nécessité d'une grande surface d'échange pour pallier le médiocre coefficient convectif des gaz.

Dans le cas d'un échangeur classique à tubes, le prix de l'échangeur croît en conséquence. Par contre, en pulvérisant des gouttes liquides dans le gaz, on est maître de la surface d'échange par le biais de la taille des gouttes. Par ailleurs, la quantité de sel liquide pulvérisée et entièrement solidifiée au déstockage, est évacuée hors du réservoir, contrairement au cas d'un échangeur classique à tube, où le sel déjà solidifié a une action néfaste sur les échanges ultérieurs. En contrepartie, deux réservoirs doivent être prévus.

Indépendamment du problème lié au changement de phase, les échangeurs gaz-particules ont déjà fait l'objet de nombreux articles [1-7], mais les résultats expérimentaux obtenus dans un cas donné sont en général difficilement transposables; il n'existe pas de modèle théorique satisfaisant permettant de traiter le problème dans toute sa généralité. Aussi, compte tenu d'un certain nombre de simplifications et d'hypothèses qui seront précisées, une méthode générale conduisant à un traitement numérique est proposée.

2. Hypothèses. - L'échangeur sera modélisé par un courant gazeux ascendant contenu dans une virole verticale cylindrique au sein duquel tombe une pluie de particules sphériques, uniformément réparties dans une section donnée de l'échangeur.

La relation entre vitesse d'injection et taille (ou taille moyenne) des gouttes est fondée sur le postulat suivant : aucune des gouttes ne doit remonter dans le sens de l'écoulement gazeux. A la limite, l'injection est donc conçue pour que la traînée qu'exerce le gaz dans l'état qui est le sien au niveau de l'injection $\left(V_{\mathrm{g}}, \rho_{\mathrm{g}}, T_{\mathrm{g}}, \mu_{\mathrm{g}}\right)$ soit exactement égale à leur poids. Le travail des forces de trainée n'entame donc pas l'énergie cinétique.

Le but de cette sujétion est d'éviter qu'un ralentissement éventuel ne fasse croître localement la concentration; dans ce cas, la croissance du nombre des chocs entre gouttes encore liquideș en surface augmenterait les coalescences.

Plus bas, dans l'échangeur, le travail de la trainée peut entamer l'énergie cinétique à condition de ne jamais l'annuler. Mais la croissance de la concentration n'a plus l'effet néfaste signalé ci-dessus. Le calcul a, d'autre part, été mené pour des concentrations volumiques relativement faibles $\left(\simeq 10^{-3}\right)$, ce qui permet de négliger l'interaction mécanique et thermique entre les gouttes. Pour de telles concentrations, il est également aisé de montrer que l'on peut négliger l'interaction des gouttes sur l'écoulement du gaz, quitte à ajouter globalement aux pertes de charge consenties pour la circulation du gaz, le terme correspondant à la perte d'énergie cinétique et potentielle des gouttes entre leur injection et leur sortie.

3. Equations. - 3.1 Equations DU MOUVEMENT D'UNE GOUTTE. - Soit $\Sigma$ la surface extérieure d'une goutte, $V$ son volume, le mouvement de son centre de gravité est donné par l'équation suivante :

$$
m \frac{\mathrm{d} \mathbf{V}}{\mathrm{d} t}=\iint_{\Sigma} \tilde{\sigma}_{\mathrm{g}} \mathbf{n} \mathrm{d} \Sigma+\iint_{\Sigma} \rho_{\mathrm{g}} g Z \mathbf{n} \mathrm{d} \Sigma m g \operatorname{grad} \mathbf{Z}_{\mathbf{g}}
$$

où :

$\tilde{\tilde{\sigma}}_{\mathrm{g}}$ représente le tenseur des contraintes modifié,

$Z_{\mathrm{g}}$ représente la cote verticale du centre de gravité, n représente la normale à la surface de la goutte, $\mathbf{T}=\iint_{\Sigma} \tilde{\tilde{\sigma}}_{\mathrm{g}} \mathbf{n} \mathrm{d} \Sigma$ représente la force de traînée, dans laquelle interviennent deux termes :

- une résistance de frottement due aux frottements visqueux sur les parois de l'obstacle, la goutte en la circonstance,

- une résistance de forme due à la différence de pression entre l'amont et l'aval de l'obstacle, résistance qui, comme son nom l'indique, est liée à la forme de l'obstacle.

Remarquons que $\rho_{\mathrm{s}} \gg \rho$, et faisons intervenir la notion de coefficient de traînée (en toute rigueur, la notion de coefficient de traînée $C_{\mathrm{x}}$ n'est applicable que pour un mouvement de translation uniforme à l'intérieur d'un fluide isotherme, ce qui n'est pas le cas ici).

L'équation de mouvement des gouttes sera donnée par :

$$
\rho_{\mathrm{s}} \frac{\mathrm{d} V_{\mathrm{s}}}{\mathrm{d} t}=\rho_{\mathrm{s}} g-\frac{3}{8} C_{\mathrm{x}} \rho \frac{U_{\mathrm{R}}^{2}}{R_{1}} .
$$


Quant au mouvement $\mathrm{du}$ gaz, il est régi par les équations suivantes :

$$
\begin{gathered}
\rho \frac{\mathrm{d} \mathbf{V}_{\mathrm{g}}}{\mathrm{d} \tau}=\rho \mathbf{F}-\operatorname{grad} P+\mu \Delta V_{\mathrm{g}}+\frac{\mu}{3} \operatorname{grad} \operatorname{div} \mathbf{V}_{\mathbf{g}} \\
\frac{\partial \rho}{\partial t}+\operatorname{div}\left(\rho \mathbf{V}_{\mathrm{g}}\right)=0
\end{gathered}
$$

En fait, en supposant les pertes de charges dans l'échangeur négligeables, la vitesse du courant gazeux vérifie la relation

$$
\stackrel{\circ}{m}_{\mathrm{g}}=\rho_{\mathrm{g}} S_{\mathrm{e}} V_{\mathrm{g}}
$$

où $\rho_{\mathrm{g}}$ est fonction uniquement de $T$.

Deux questions restent cependant sans réponse :

3.1.1 Les gouttes que l'on forme restent-elles dans l'état géométrique initial ?

3.1.2 Quel est le coefficient de traînée, compte tenu de leur déformation éventuelle ?

3.1.1 Pour tenter d'éclairer le premier point, deux phénomènes sont à considérer. - Le risque de coalescence entre gouttes. Ce risque étant difficilement quantifiable, mais restant vraisemblablement faible, compte tenu des concentrations, il n'en sera pas tenu compte dans la suite de l'exposé. Il est nécessaire de vérifier, par ailleurs, que les gouttes, formées de manière à satisfaire le critère énoncé précédemment, sont stables et ne se subdivisent pas. Les résultats s'expriment en fonction du nombre de Weber $W e=\rho_{\mathrm{g}} U_{\mathrm{R}}^{2} R_{1} / A$ où $A$ représente la tension superficielle de la goutte [11]. Le nombre de Weber traduit le rapport de deux effets contraires :

La tension superficielle qui génère une pression assurant la cohésion de la goutte, et les forces aérodynamiques qui, par la création d'une circulation du liquide à l'intérieur de la goutte, engendrent une pression dynamique qui tend à la faire éclater; si la valeur de la pression dynamique dépasse la valeur de la pression due à la tension superficielle, il y a rupture de l'interface.

La tension superficielle du $\mathrm{NaCl}$, exprimée en dyne/cm, est donnée par la formule suivante : $A=216,21-0,0930$ T [10].

Pour une goutte de rayon $R=0,5 \mathrm{~mm}$, de vitesse $5 \mathrm{~m} / \mathrm{s}$, et de température initiale de $850^{\circ} \mathrm{C}$, le nombre de Weber entre l'injection et la formation de la goutte, est compris dans l'intervalle suivant :

$$
1,99 \leqslant W e \leqslant 2,07,
$$

alors que la valeur limite est de l'ordre de 5 . Il semble donc bien que les risques d'éclatement soient faibles, d'autant plus que ce processus n'est pas instantané et nécessite une certaine longueur de parcours ou un temps de retard pendant lequel la génération d'une croûte solide superficielle tend à s'opposer à la division. De ce fait, la stabilité sera d'autant meilleure que l'injection du liquide sera faite à une température proche de la température de fusion.
3.1.2 Choix du coefficient de traînée. - Compte tenu des cycles qui ont été retenus, le nombre de Reynolds des gouttes se trouvera dans un domaine tel que $50 \leqslant R e \leqslant 1000$. Il n'est donc plus possible d'employer la classique formule de Stockes. De nombreuses formules généralement empiriques couvrent ce domaine. Citons :

$$
\begin{array}{rlrl}
2 & \leqslant R e \leqslant 500 & C_{\mathrm{x}}=18,5 R e^{-0,6} \\
10 \leqslant R e \leqslant 10^{3} & C_{\mathrm{x}}=13 / \sqrt{R e} \\
1 \leqslant \operatorname{Re} \leqslant 400 & C_{\mathrm{x}}=\frac{24}{\operatorname{Re}}\left(1+\frac{R e^{2 / 3}}{6}\right)
\end{array}
$$

(formule de Klyachko)

$$
0 \leqslant R e \leqslant 2 \times 10^{5} \quad C_{\mathrm{x}}=\frac{24}{\operatorname{Re}}+\frac{6}{1+\sqrt{\operatorname{Re}}}+0,4
$$

(formule de White).

Le choix de l'une ou l'autre de ces formules, sans être critique, peut conduire à des différences sensibles dans la prévision du comportement des particules. Dans la suite de l'exposé, la première relation a été choisie pour présenter quelques résultats numériques.

3.2 EQUATIONS RÉGISSANT LE TRANSFERT THERMIQUE. - Dans l'étude du phénomène de solidification des gouttes, les hypothèses suivantes ont été faites :

a) Les gouttes sont parfaitement sphériques.

b) Le transfert radiatif est négligé, approximation justifiée dans le cas de l'air, mais qui risque de l'être beaucoup moins dans le cas du $\mathrm{CO}_{2}$. Cependant, l'absence de propriétés radiatives concernant les sels fondus, rend impossible toute tentative de calcul.

c) Le coefficient convectif est pris constant sur toute la surface et égal au coefficient convectif moyen. (Cette hypothèse se justifie dans la mesure où les gouttes sont soumises à un mouvement de rotation qui tend à moyenner le coefficient local.) Ceci permet de conserver l'hypothèse d'une symétrie sphérique pour la croissance de la croûte.

d) Les données thermiques, physiques et mécaniques de la goutte sont indépendantes de la température. En particulier, dans les résultats qui suivent, on a supposé la masse volumique de la phase solide, égale à la masse volumique de la phase liquide. Mais il serait également possible de tenir compte de cette variation de masse volumique en admettant qu'il se forme au centre de la goutte un trou à symétrie sphérique. Des résultats expérimentaux sont entrepris afin d'affiner le modèle à ce point de vue.

e) On pourrait également envisager entre l'intérieur liquide de la goutte et sa paroi solide un transfert par convection naturelle qui améliorerait le transfert conductif seul pris en compte ici.

En fait, en cas de surchauffe du liquide, il y a une différence de température qui s'amoindrit avec le temps entre le liquide et la paroi à $T_{\mathrm{F}}$. Mais la longueur 
caractéristique de l'ordre de $1 \mathrm{~mm}$ introduit un facteur de $10^{-9}$ dans le Grashof. Comme par ailleurs $V^{2}$ est très élevé au voisinage de l'état solide, le Grashof reste très faible et la convection naturelle dans la goutte négligeable.

3.2.1 Choix du Nusselt. - Les nombres de Nusselt sont en général déterminés pour une sphère absolument fixe dans un écoulement monodimensionnel en moyenne. Or ici, les gouttes sont elles-mêmes en mouvement dans un écoulement susceptible de posséder une intensité de turbulence notable, ce qui rend le problème beaucoup plus complexe. Cependant, en l'absence de renseignements sur ces phénomènes dont l'étude n'a pas été réalisée de manière systématique [5], on prendra une expression valable pour une sphère immobile, et qui tend donc à minimiser la valeur de $h$.

D'autre part, on peut se demander en quoi l'anisothermie goutte-gaz qui implique une convection naturelle modifie le champ des vitesses du gaz et, partant, la valeur du Nusselt et du coefficient de traînée.

En fait, l'influence de la convection naturelle sera d'autant plus appréciable que $G r / R e^{2}$ sera grand. Dans [9], est fixée une valeur de $G r / R e^{2}$ égale à 1,67 , au-dessus de laquelle il n'est plus possible de négliger la valeur de la convection naturelle. Un calcul fait dans le cas des exemples cités dans la suite de l'exposé permet de trouver des valeurs de l'ordre de $10^{-4}$ à $10^{-5}$ ce qui exclut totalement ce phénomène.

Il sera donc employé une formule établie par B. D. Katsnel et F. A. Timofeyera [10] dans le cas où $100 \leqslant R e \leqslant 10^{5}$.

$N u=2+0,03 \operatorname{Pr}^{0,33} \operatorname{Re}^{0,54}+0,35 \operatorname{Pr}^{0,356} \operatorname{Re}^{0,58}$.

3.2.2 Choix du modèle de cristallisation.3.2.2.1 Mise en équation du problème. - Moyennant les hypothèses précédentes, et en supposant que le refroidissement de la goutte est uniquement conductif, on obtient le système d'équations suivant :

$$
\begin{aligned}
\frac{a}{R} \frac{\partial^{2}(R T)}{\partial R^{2}} & =\frac{\partial T}{\partial t} \\
\lambda_{\mathrm{s}}\left(\frac{\partial T_{\mathrm{s}}}{\partial R}\right)_{R=R_{\mathrm{F}}} & =\rho_{\mathrm{s}} L \frac{\mathrm{d} R_{\mathrm{F}}}{\mathrm{d} t}+\lambda_{\mathrm{L}}\left(\frac{\partial T_{\mathrm{L}}}{\partial R}\right)_{R=R_{\mathrm{F}}} \\
-\lambda_{\mathrm{s}}\left(\frac{\partial T_{\mathrm{s}}}{\partial R}\right)_{R=R_{1}} & =h\left(T_{\mathrm{s}}\left(R=R_{1}\right)-T_{\mathrm{g}}\right) \\
T\left(t, R_{\mathrm{F}}\right) & =T_{\mathrm{F}}
\end{aligned}
$$

( $a=a_{\mathrm{L}}$ dans la phase liquide),

( $a=a_{\mathrm{s}}$ dans la phase solide).

Compte tenu du fait que l'on s'intéresse à des gouttelettes petites $(0,5 \leqslant R \leqslant 1 \mathrm{~mm})$ et que le rapport $C_{\mathrm{pl}} / L$ est très petit pour $\mathrm{CINa}$, on peut poser que toute l'énergie stockée sous forme de chaleur sensible du liquide est pratiquement déstockée lorsque commence le processus de solidification superficielle. Autrement dit, la goutte est quasi-isotherme à un niveau qui, lui, dépend du temps quand la chaleur latente commence à intervenir.

Il est courant et commode, pour les résolutions analytiques, d'écrire le système ci-dessus en variables adimensionnées. Ce type de résolution n'est utilisé, comme dans de nombreux articles antérieurs [14-17], que dans le cas où $T_{\mathrm{L}}=T_{\mathrm{F}}$.

Néanmoins, la référence de température est délicate à définir. On devrait prendre la différence entre la température locale du gaz et la température de fusion. On peut choisir la valeur maximale de cette quantité, à savoir $T_{\mathrm{F}}-T_{\mathrm{ge}}$. Dans ce cas :

$$
\begin{aligned}
\frac{1}{r} \frac{\partial^{2}\left(r T^{+}\right)}{\partial r^{2}} & =\frac{\partial T^{+}}{\partial \tau} \\
\frac{\mathrm{d} r_{\mathrm{F}}}{\mathrm{d} \tau} & =S t e\left(\frac{\partial T^{+}}{\partial r}\right)_{r=r_{\mathrm{F}}} \\
\left(\frac{\partial T^{+}}{\partial r}\right)_{r=1} & =-B i\left(T_{(r=1)}^{+}-T_{\mathrm{g}}^{+}\right) \\
T^{+}\left(t, r_{\mathrm{F}}\right) & =0
\end{aligned}
$$

équations qui font apparaître les nombres de Biot et de Stephan

$$
\begin{aligned}
B i & =\frac{h R_{1}}{\lambda_{\mathrm{s}}} \\
\text { Stephan } & =\frac{C_{\mathrm{ps}}\left(T_{\mathrm{F}}-T_{\mathrm{ge}}\right)}{L} .
\end{aligned}
$$

Elles ont l'intérêt d'être d'un emploi très facile et peuvent donc être intégrées dans un schéma de calcul plus complexe, comme par exemple celui du calcul d'un échangeur gaz-gouttes. Ces modèles s'appliquent d'autant mieux que le nombre de Stéphan est petit, mais deviennent en général complètement inadaptés lorsque $R_{F} \rightarrow 0$, ce qui n'est pas très important puisque, même en l'absence de contraction, l'énergie est presque complètement déstockée pour $R_{\mathrm{F}}=0,2 R_{1}$; si l'on tient compte de la contraction à partir du rayon extérieur, le front de solidification $n$ 'atteint jamais le centre mais en admettant la variation de $\rho$ de $15 \%$ de la valeur initiale, liquide $R_{\mathrm{F}}=0,5 R_{1}$ si $t \rightarrow+\infty$.

Les modèles numériques présentent l'avantage de s'adapter à la quasi-totalité des valeurs du nombre de Stephan et de rester exacts lorsque $\boldsymbol{R}_{\mathrm{F}} \rightarrow 0$. Ils nécessitent cependant un temps calcul plus important que les schémas analytiques et sont d'une mise en œuvre plus délicate.

Deux programmes ont été réalisés :

- l'un utilise un schéma aux différences finies, limité à une seule taille de goutte, mais permet de prendre en compte le déstockage de la chaleur sensible avant le début de la cristallisation et après la fin de celle-ci (une plus ample description de ce schéma est donnée dans le paragraphe suivant); 
- l'autre exploite un schéma analytique (développement parabolique de la température), suivi d'un schéma numérique qui peut prendre en compte le déstockage de la chaleur sensible après la solidification et non pas avant, ce qui implique une température initiale peu différente de $T_{\mathrm{f}}$. Cette restriction est compensée par la possibilité de traiter une répartition quelconque mais donnée des gouttes.

3.2.2.2 Schéma numérique aux différences finies. - Le programme a été limité à une seule taille de gouttes, pour des raisons de temps de calcul, mais son extension à une répartition discrète en taille de gouttes ne poserait aucun problème.

Compte tenu des approximations qui ont été faites (paragraphe précédent), un schéma relativement simple mais satisfaisant du point de vue précision a été recherché, ce qui conduit à utiliser le principe exposé par Bonacina et col. dans [12] et qui consiste à représenter la chaleur latente de changement d'état par un saut de capacité calorifique sur un intervalle $2 \varepsilon$. On aboutit alors à la résolution de l'équation de la chaleur

où

$$
\rho C_{\mathrm{p}}^{*} \frac{\partial T}{\partial t}=\frac{1}{R^{2}} \frac{\partial}{\partial R}\left(\lambda R^{2} \frac{\partial T}{\partial R}\right)
$$

$$
C_{\mathrm{p}}^{*}=\left\{\begin{array}{l}
C_{\mathrm{ps}}(T) \text { pour } T<T_{\mathrm{F}}-\varepsilon \\
C_{\mathrm{pl}}(T) \text { pour } T>T_{\mathrm{F}}+\varepsilon \\
\frac{L}{2 \varepsilon}+\frac{C_{\mathrm{ps}}\left(T_{\mathrm{F}}\right)+C_{\mathrm{pl}}\left(T_{\mathrm{F}}\right)}{2} \\
\text { pour } T_{\mathrm{F}}-\varepsilon \leqslant T \leqslant T_{\mathrm{F}}+\varepsilon .
\end{array}\right.
$$

La résolution numérique du système d'équations discrétisé a été effectuée au moyen d'un schéma de Crank-Nicholson, qui conduit à la résolution d'un système matriciel du type :

$$
A^{n+1} T^{n+1}=B^{n} T^{n}+C^{n+1}
$$

où $A^{n+1}$ et $C^{n+1}$ dépendent du nouveau champ de température. La résolution du système a été effectuée à l'aide de la méthode itérative de Gauss-Seidel. Un tel schéma permettant de prendre en compte les variations de $\lambda$ en fonction de la température mais pour des raisons de temps-calcul, les résultats de la suite de l'exposé ont été obtenus pour $\lambda$ constant, cette approximation étant d'ailleurs très largement suffisante, compte tenu des hypothèses faites sur les variations de masse volumique et l'incertitude sur les valeurs de $\lambda$ à la solidification.

Dans le cas où le liquide est initialement à température de fusion, la comparaison avec d'autres schémas du type perturbation montre que ce schéma aux différences finies décrit de manière parfaitement satisfaisante le processus de solidification d'une sphère tout en présentant l'intérêt d'être encore applicable lorsque la sphère est entièrement solidifiée.

3.2.2.3 Résolution du système d'équations complet. - La résolution du système est présentée dans le cas de gouttes monodimensionnelles mais sa généralisation au cas d'une répartition de gouttes est immédiate.

Le fait que l'échangeur est intégré dans un cycle thermodynamique fixe un certain nombre de données qui peuvent se regrouper dans un premier lot :

a) la température d'entrée du gaz,

b) la température de sortie du gaz,

c) le débit de gaz,

d) la température d'entrée du $\mathrm{NaCl}$ liquide donc supérieure à $801^{\circ} \mathrm{C}$,

e) la température de sortie moyenne du $\mathrm{NaCl}$, autrement dit l'énergie résiduelle de la goutte,

$f$ ) le débit $\stackrel{\circ}{m}_{\text {s de }} \mathrm{NaCl}$.

Dans un deuxième groupe, il reste huit paramètres qui définissent l'échangeur mais que le cycle thermodynamique aval n'impose pas. Ce sont :

a) la vitesse d'injection $V_{\text {si }}$ des gouttes,

b) la vitesse de sortie des gouttes,

c) le rayon des gouttes,

d) la concentration volumique initiale,

e) le temps de séjour des gouttes,

f) le rayon de l'échangeur,

g) la longueur de l'échangeur,

h) l'état des gouttes dans la section finale.

Entre ces différents paramètres existent des relations :

$$
\begin{aligned}
& \stackrel{\circ}{m}_{\mathrm{s}}=\rho_{\mathrm{s}} \pi R_{\mathrm{c}}^{2} C_{\mathrm{v}} V_{\mathrm{si}} \\
& R_{1}=\frac{3}{8} \frac{\rho_{\mathrm{g}}}{\rho_{\mathrm{s}}} C_{\mathrm{x}} \frac{\left(V_{\mathrm{s}}+V_{\mathrm{g}}\right)_{\mathrm{i}}}{g}
\end{aligned}
$$

donnant le rayon à imposer aux gouttes en fonction de la vitesse d'injection de telle sorte que l'interversion éventuelle de $a$ et de $c$ dans les deux paramètres d'entrée est immédiate

$$
\rho_{\mathrm{s}} \frac{\mathrm{d} V_{\mathrm{s}}}{\mathrm{d} t}=\rho_{\mathrm{s}} g-\rho \frac{\left(V_{\mathrm{g}}+V_{\mathrm{s}}\right)^{2}}{R_{1}} f(R e)
$$

donnant la vitesse des gouttes

$$
\frac{\mathrm{d} X}{\mathrm{~d} t}=V_{\mathrm{s}}
$$

déterminant, avec la longueur de l'échangeur, conséquence elle-même de l'échange thermique, le temps de séjour des gouttes.

$$
\frac{\mathrm{d} Q}{\mathrm{~d} t}=\varphi=f\left(h, T_{\mathrm{g}}, t\right)
$$

définissant par intégration sur le temps l'état thermique des gouttes.

$$
\stackrel{\circ}{m}_{\mathrm{g}} C_{\mathrm{pg}} \frac{\mathrm{d} T_{\mathrm{g}}}{\mathrm{d} t}=-N \varphi
$$


loi d'évolution de la température du gaz, qui permet donc de déterminer la longueur de l'échangeur.

Enfin, les calculs supposent connues par ailleurs les lois $N u=f(R e, \operatorname{Pr}) C_{\mathrm{x}}=f(R e)$. Voir courbes 10 et 11 .

En tout instant et en tout endroit dans l'échangeur, le Reynolds est caractérisé par le diamètre de la goutte et la vitesse relative gaz-gouttes à l'endroit considéré.

Finalement, on obtient six relations reliant entre eux les huit paramètres; deux des paramètres sont donc à choisir arbitrairement. Dans la suite de l'exposé, les concentrations volumiques initiales et la vitesse d'injection $V_{\text {si }}$ sont fixées $a$ priori, mais tout autre choix peut être fait.

Le système d'équations ainsi obtenu est soluble sans difficulté par une méthode de type Adams. Dans les différents schémas numériques utilisés, les données concernant le gaz ont été obtenues par une méthode d'interpolation à Newton, à partir de valeurs discrètes sur le domaine de température utilisé dans le calcul.

Un organigramme du programme utilisant le schéma de cristallisation aux différences finies est donné $\mathrm{F} 1$.

Avant de présenter deux exemples d'application et leurs résultats, quels commentaires et critiques peut-on apporter au principe de résolution qui vient d'être exposé ?

Ils sont de trois ordres :

a) Les lois de $C_{\mathrm{x}}(R e)$ et de $N u(R e)$ sont fondées sur des expériences de convection forcée assez éloignées des conditions d'utilisation présentes. Ce fait a déjà été discuté en 3.2.1.

b) L'écoulement gazeux est typiquement turbulent. Les échelles et les temps caractéristiques sont-ils suffisants pour perturber sensiblement l'écoulement supposé vertical comme il le serait en régime laminaire ? En particulier, on peut craindre que la turbulence n'induise des fluctuations de concentration plus ou moins instable dans toute la hauteur de l'échangeur, et surtout une variation de la concentration en fonction du rayon dans une même section perpendiculaire à l'axe.

Des calculs sont actuellement entrepris pour juger de l'importance du phénomène mais une réponse décisive ne pourra sans doute être apportée que par les expériences qui vont être menées incessamment.

c) La sphéricité de la croûte formée aura une influence sans doute modérée, mais non négligeable sur les transferts thermiques. Cette sphéricité ne peut être assurée en toute rigueur que si la goutte est soumise à un mouvement de rotation. Là encore, seule l'expérience pourra fournir les renseignements nécessaires au développement d'un modèle plus précis.

4. Annexe. - 4.1 Exemples de Calculs. - Deux calculs d'échangeurs dont les données sont prises de manière à permettre leur intégration dans un cycle thermodynamique de production d'électricité de $10 \mathrm{MW}$ sont présentés. Les valeurs sont déterminées pour deux options du circuit aval (turbine à gaz, compresseur) calculées par d'autres équipes travaillant sur le projet THEM (E.D.F., C.E.A.).

Les caractéristiques communes aux deux échangeurs sont les suivantes : Le gaz utilisé est de l'air à 20 bar, en circuit fermé, la puissance thermique échangée est de $25 \mathrm{MW}$.

La vitesse d'injection et la concentration volumique initiales ont été fixées arbitrairement à $2 \mathrm{~ms}^{-1}$ et $10^{-3}$ respectivement. Les six autres paramètres en découlent. Un schéma général (F 2) de l'installation complète est donné avec les valeurs particulières ressortant de deux options différentes pour l'utilisation aval.

\subsection{VALEURS PARTICULIÈRES AU PREMIER CALCUL. -} 4.2.1 Considérations générales. - Outre celles communes aux deux calculs et qui ont été citées précédemment, les caractéristiques imposées de l'échangeur sont les suivantes : les températures d'entrée et de sortie du gaz sont de $508,4^{\circ} \mathrm{C}$ et de $800^{\circ} \mathrm{C}$ respectivement; le débit est de $75,9 \mathrm{~kg} / \mathrm{s}$. La température d'entrée du $\mathrm{NaCl}$ est de $851^{\circ} \mathrm{C}$, la température de sortie moyenne de $550^{\circ} \mathrm{C}$, le débit est de $30,6 \mathrm{~kg} / \mathrm{s}$.

La température d'entrée de $851^{\circ} \mathrm{C}$ implique une part de fonctionnement en chaleur sensible, fait qui tend à remonter le niveau thermique du gaz pour une même différence de température gaz-gouttes dans la section d'injection. Le rendement d'une turbine à gaz est grandement favorisé par une élévation de température à ces niveaux.

4.2.2 Résultats et interprétation des courbes. Le calcul permet d'aboutir aux résultats suivants :

Le rayon des gouttes prises monodimensionnelles est de $0,706 \mathrm{~mm}$, leur vitesse de sortie est de $1,86 \mathrm{~m} / \mathrm{s}$, leur temps de séjour est de 5,4 s. Le rayon de l'échangeur est de $1,774 \mathrm{~m}$, sa longueur est de $10,5 \mathrm{~m}$. Le coefficient convectif moyen h est de $770 \mathrm{~W} / \mathrm{m}^{2}{ }^{\circ} \mathrm{C}$, la surface d'échange par $\mathrm{kg}$ de sel est de $2,74 \mathrm{~m}^{2} / \mathrm{kg}$.

Les conséquences de la conception sont intéressantes à considérer et à comparer avec ce que nécessite un échangeur classique. La surface d'échange présente en permanence dans l'échangeur, et produit de la surface d'échange par $\mathrm{kg}$ de sel, par le temps de séjour et par le débit de sel, remplace une surface d'échangeur à tube de $450 \mathrm{~m}^{2}$. En fait, si l'on revient aux valeurs de $U$ (coefficient d'échange global) plus réalistes $(U \simeq 250$ à 300$)$, c'est $1150 \mathrm{~m}^{2}$ qui seraient nécessaires dans le cas de l'échangeur à tubes.

Des courbes ont été tracées de manière à détailler le fonctionnement de l'échangeur. L'allure de la courbe 1 montre que le critère $R_{1}=f\left(V_{\mathrm{si}}\right)$ défini dans les pages précédentes, conduit à une vitesse de sel, donc à une concentration volumique relativement stable dans l'échangeur. La courbe 2 illustre la quasiconstance du coefficient convectif gaz-gouttes $h$, tout au long de l'échangeur. Les variations de la 


\section{Organigramme}

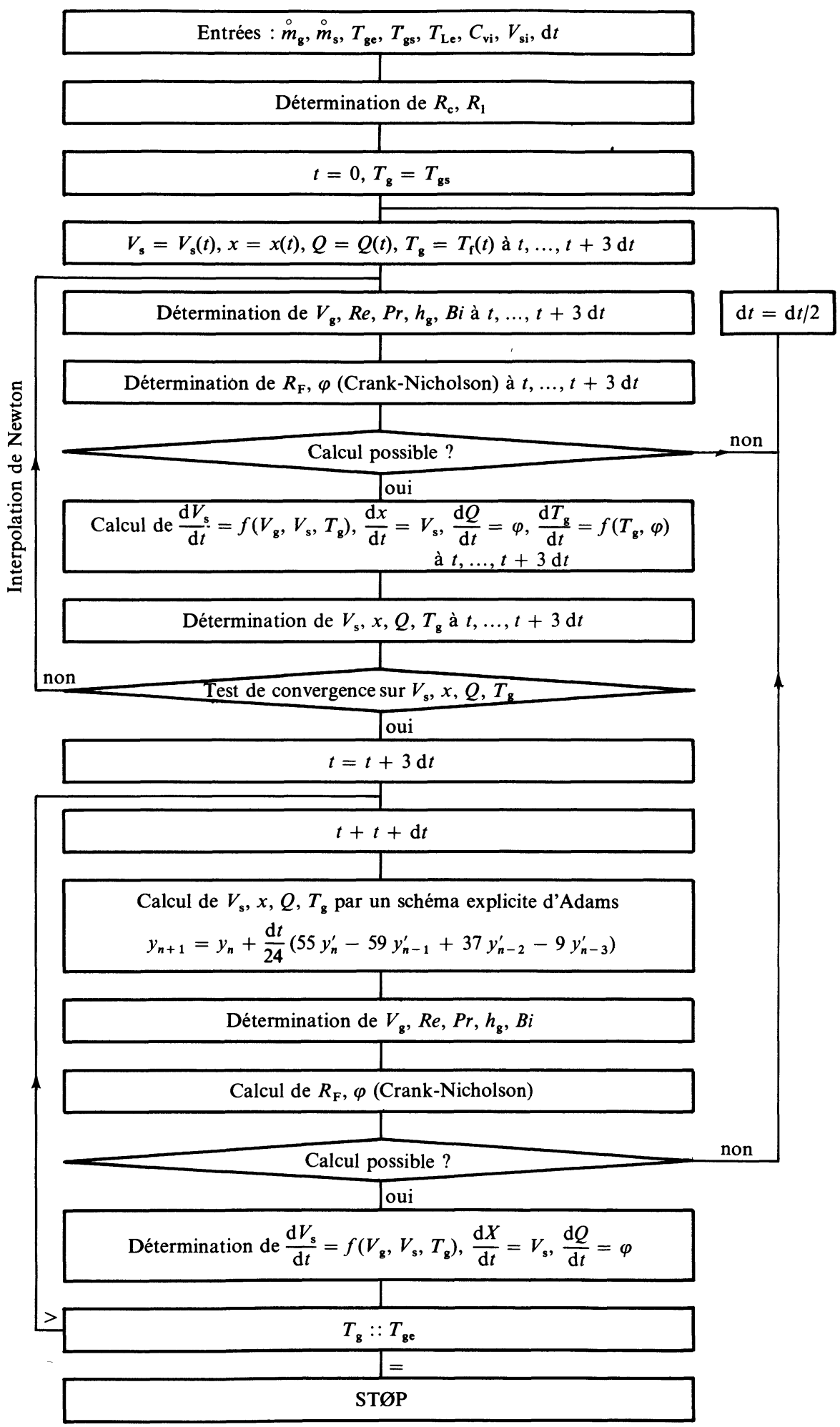

viscosité cinématique du gaz en fonction de $T$ compensent donc à peu près la variation de $\rho$.

Dans l'équation $\frac{\mathrm{d} T_{\mathrm{g}}}{\mathrm{d} x}=\frac{-3 \eta h\left(T\left(R=R_{1}\right)-T_{\mathrm{g}}\right)}{\rho_{\mathrm{s}} V_{\mathrm{s}} C_{\mathrm{pg}} R_{1}}$

le terme $\frac{3 \eta h}{\rho_{\mathrm{s}} V_{\mathrm{s}} C_{\mathrm{pg}} R_{1}}$ varie donc très peu suivant $x$.
L'échange de chaleur gaz-gouttes est essentiellement conditionné par l'écart de température entre la surface des gouttes et le gaz. Ceci explique l'allure de la courbe 3 qui représente le flux extrait d'une goutte en fonction du temps ou en fonction de l'abscisse dans l'échangeur. Elle traduit l'évolution de l'écart 

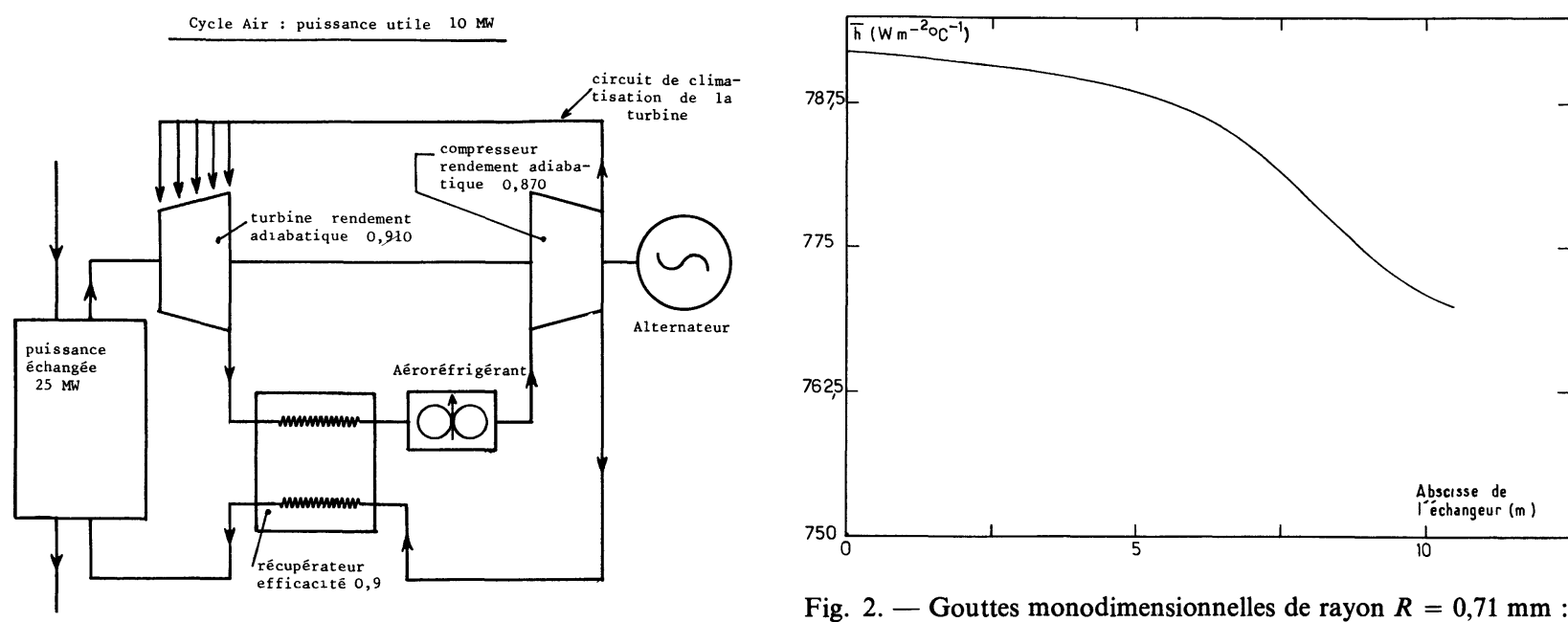

Fig. 2. - Gouttes monodimensionnelles de rayon $R=0,71 \mathrm{~mm}$ : Evolution du coefficient convectif moyen gaz-gouttes en fonction de l'abscisse dans l'échangeur.

\begin{tabular}{|c|c|c|}
\cline { 2 - 3 } \multicolumn{1}{c|}{} & Option 1 & Option 2 \\
\hline Débit d'air & $75,9 \mathrm{~kg} / \mathrm{s}$ & $120 \mathrm{~kg} / \mathrm{s}$ \\
\hline $\begin{array}{c}\text { Temperature air à 1'entrée de } \\
\text { 1'échangeur }\end{array}$ & $508^{\circ} \mathrm{C}$ & $597^{\circ} \mathrm{C}$ \\
\hline $\begin{array}{c}\text { Température air à la sortie de } \\
\text { 1'échangeur }\end{array}$ & $800^{\circ} \mathrm{C}$ & $781^{\circ} \mathrm{C}$ \\
\hline Pression air & $20 \mathrm{bar}$ & $20 \mathrm{bar}$ \\
\hline Débit Na Cl & $30,6 \mathrm{~kg} / \mathrm{s}$ & $48,4 \mathrm{~kg} / \mathrm{s}$ \\
\hline Température entrée Na Cl & $851^{\circ} \mathrm{C}$ & $801^{\circ} \mathrm{C}$ \\
\hline Température de sortie moyenne & $550^{\circ} \mathrm{C}$ & $801^{\circ} \mathrm{C}$ \\
\hline Na C1 & $25 \mathrm{MW}$ & $25 \mathrm{MW}$ \\
\hline Puissance thermique & $10 \mathrm{MW}$ & $10 \mathrm{MW}$ \\
\hline
\end{tabular}

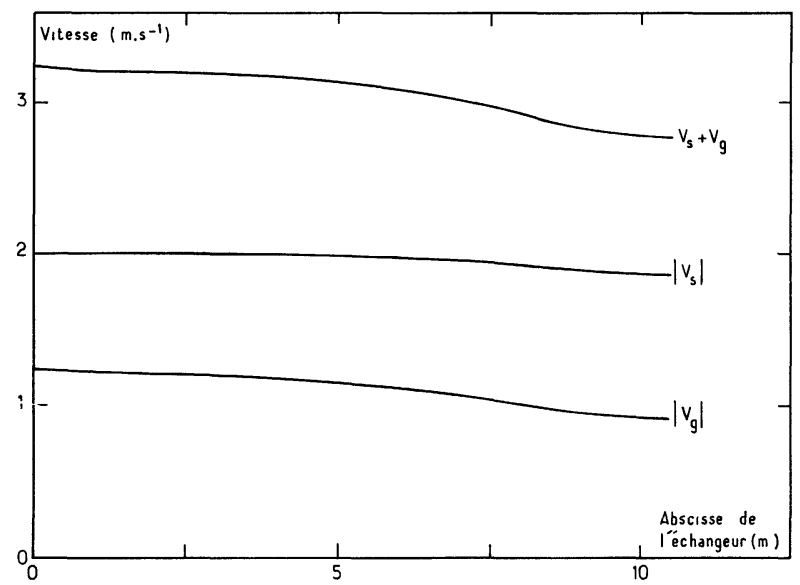

[One drop size : $R=0.71 \mathrm{~mm}$. Variations of the mean heat transfer coefficient with distance along the exchanger.]

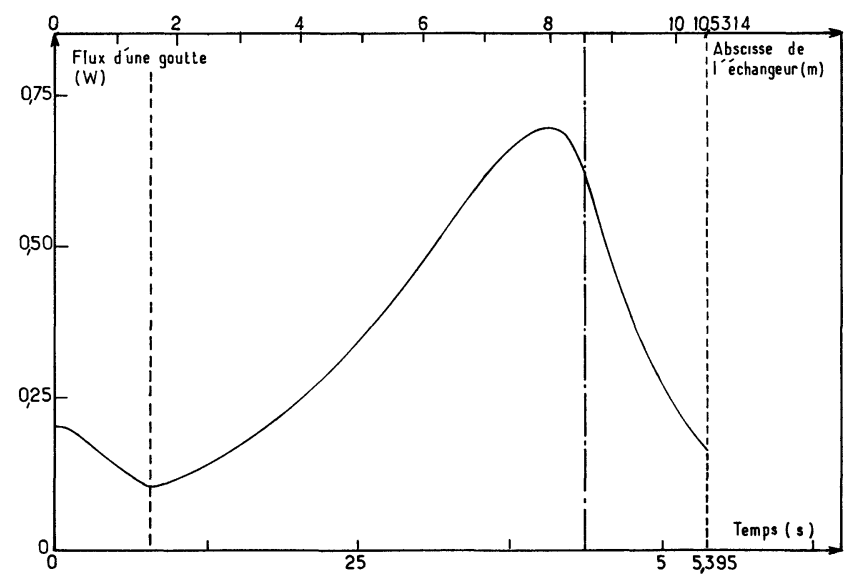

Fig. 3. - Gouttes monodimensionnelles de rayon $R=0,71 \mathrm{~mm}$ : Flux d'une goutte en fonction du temps ou en fonction de l'abscisse dans l'échangeur.

[One drop size : $R=0.71 \mathrm{~mm}$. Variations of the heat transfer from a drop with absciss or with time.]

Fig. 1. - Gouttes monodimensionnelles de rayon $R=0,71 \mathrm{~mm}$ : Expression de la vitesse du gaz, de la vitesse des gouttes, de la vitesse relative gaz-gouttes en fonction de l'abscisse dans l'échangeur, la section d'injection étant prise comme origine.

[One drop size : $R=0.71 \mathrm{~mm}$. Variations of velocities of gas, drops and of the relative velocity with distance along the exchanger. The entering section of the drops is the origin.]

de température gaz-gouttes représenté par la figure 4 : peu de temps après l'injection, les gouttes ne déstockent que la chaleur sensible du sel liquide. Les gouttes

étant alors grosso modo isothermes, on peut dire que l'écart de température $T_{\mathrm{p}}-T_{\mathrm{g}}$ dépend de la valeur du rapport $\frac{\stackrel{\circ}{\mathrm{s}}_{\mathrm{s}} C_{\mathrm{pl}}}{\stackrel{\circ}{m}_{\mathrm{g}} C_{\mathrm{pg}}}=0,4$. Ce rapport est inférieur à 1 et l'écart de température goutte-air décroît, ainsi que le flux cédé.

Quand la température de surface $T_{\mathrm{p}}$ atteint la température de solidification $T_{\mathrm{F}}$, une croûte solide commence à se former avec déstockage simultané en chaleur sensible et latente. Le flux dégagé par le déstockage de la chaleur latente étant considérable relativement à la chaleur sensible extraite, la température gaz-gouttes croît et le flux cédé par la goutte rayon de solidification dans la goutte reste proche du rayon extérieur. En conséquence, l'écart de tempé- 


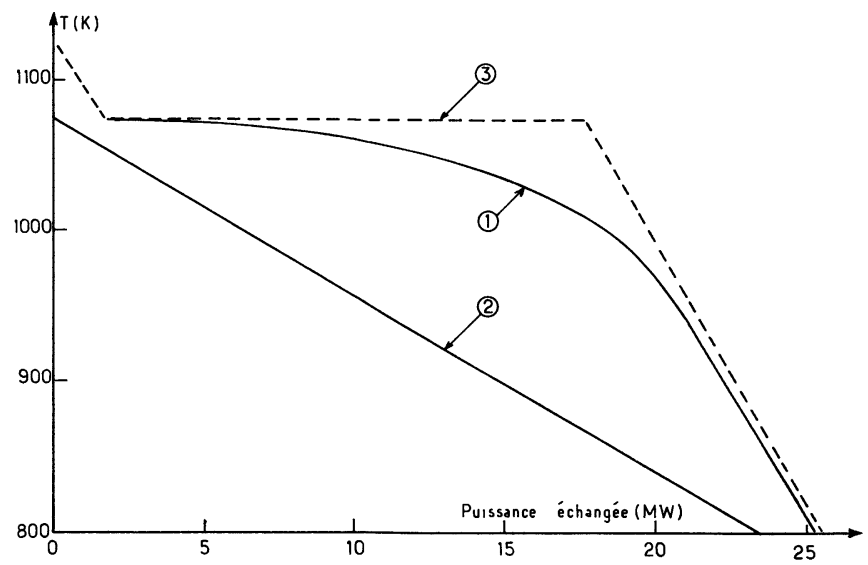

Fig. 4. - Gouttes monodimensionnelles de rayon $R=0,71 \mathrm{~mm}$ : 1. - Température de surface d'une goutte de sel. 2. - Température de l'air. 3. - Température théorique de la goutte s'il n'y avait pas de chute de température dans la croûte. Ces trois courbes sont tracées en fonction de la puissance échangée depuis la section d'injection.

[One drop size : $R=0.71 \mathrm{~mm}$. 1. - Surface temperature of a drop. 2. - Temperature of the gas. 3. - Theoretical temperature of a drop without temperature drop in the solid phase. As a function of the power exchanged since the entering section of the drops.]

rature gaz-goutte croît et le flux cédé par la goutte croît aussi.

Quand la goutte est entièrement solide, elle ne déstocke plus que de la chaleur sensible du solide. Mais, comme $\frac{\stackrel{\circ}{\mathrm{s}}_{\mathrm{s}} C_{\mathrm{ps}}}{\dot{\circ}_{\mathrm{g}} C_{\mathrm{pg}}}=0,35$ à une longueur et à un accroissement de température du gaz donnés correspondra une diminution beaucoup plus importante de la température moyenne de la goutte si bien que l'écart de température $T_{\mathrm{p}}-T_{\mathrm{g}}$ diminue avec le flux échangé.

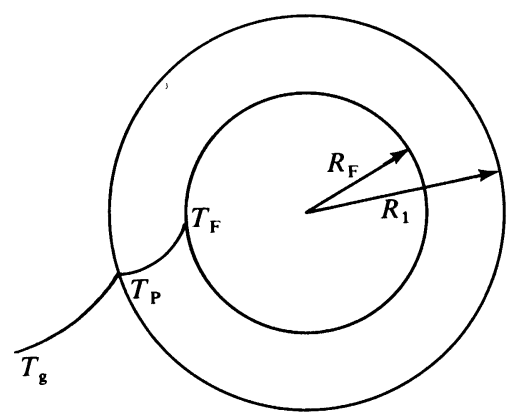

Sur la figure 4 , on représente à titre comparatif la température d'une goutte où l'on négligerait toute chute de température dans la croûte solide. La comparaison montre que la chute de température dans la croûte est maximale à la fin du déstockage de la chaleur latente.

La courbe 5 relie l'abscisse de la goutte dans l'échangeur à la quantité de chaleur cédée depuis l'injecteur. On remarque bien entendu que la phase de déstockage de la chaleur latente correspond à la portion de l'échangeur la plus efficace.

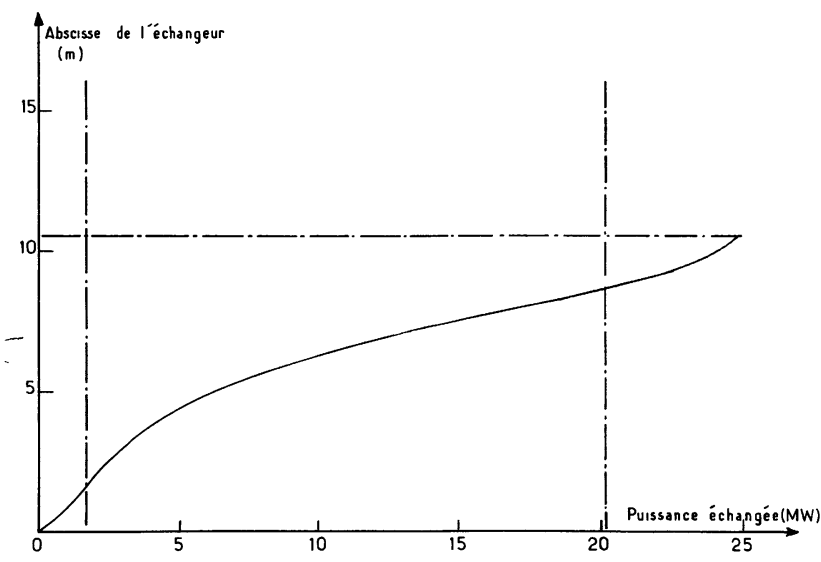

Fig. 5. - Gouttes monodimensionnelles de rayon $R=0,71 \mathrm{~mm}$ : Relation liant l'abscisse dans l'échangeur à la puissance échangée depuis la section d'entrée des gouttes.

[One drop size : $R=0.71 \mathrm{~mm}$. Absciss in the exchanger as a function of the power exchanged since the entering section of the drops.]

4.3 Deuxième Calcul. Valeurs particulières IMPOSÉES POUR UNE DEUXIÈME OPTION DU CYCLE AVAL. - 4.3.1 Considérations générales. - C'est un échangeur destiné à un cycle fermé avec récupération du type $\mathrm{F} 2$. Les caractéristiques imposées à l'échangeur sont les suivantes : la température d'entrée du gaz est de $597^{\circ} \mathrm{C}$, sa température de sortie de $781{ }^{\circ} \mathrm{C}$, son débit est de $120 \mathrm{~kg} / \mathrm{s}$.

Deux cas ont été calculés; l'un où les gouttes sont monodimensionnelles, caractérisées par $R_{0}$; l'autre où il existe une répartition discrète et symétrique de gouttes suivant une courbe voisine d'une courbe de Gauss, et dont l'allure est donnée en 6. A chaque taille de gouttes correspond un numéro $1,2, \ldots 11$ commun à $6,7,8$; la température d'entrée du $\mathrm{NaCl}$ est de $801{ }^{\circ} \mathrm{C}$ et le débit du sel $(48,4 \mathrm{~kg} / \mathrm{s})$

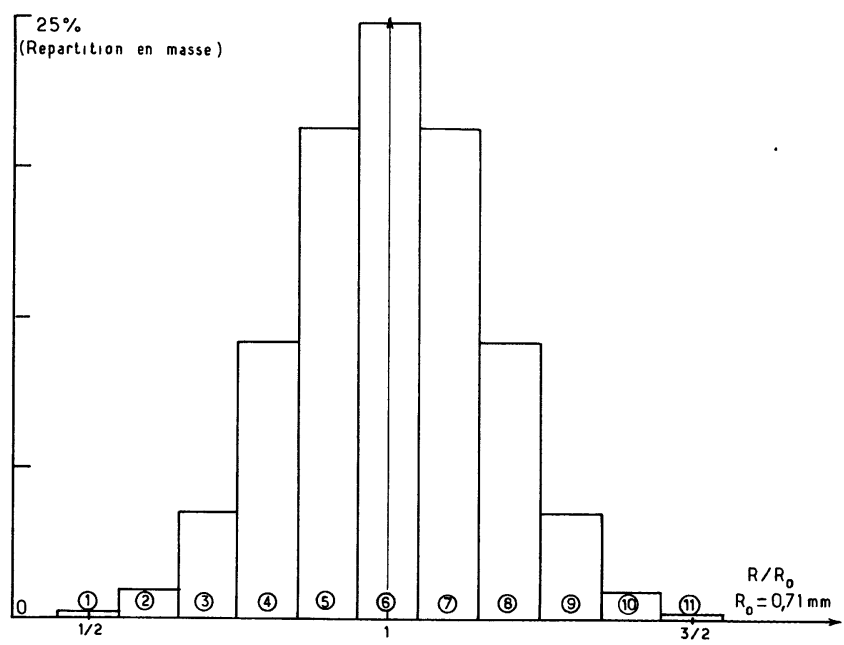

Fig. 6. - Cas d'une répartition de gouttes : Répartition relative en masse des gouttes suivant le rayon.

[Distribution of drops : Mass distribution of drops as a function of the radius.] 


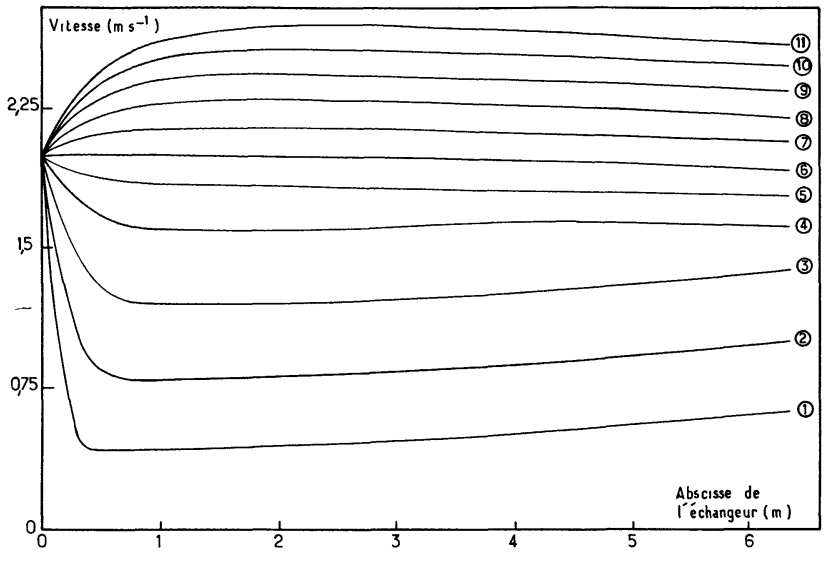

Fig. 7. - Cas d'une répartition de gouttes : Vitesse des gouttes suivant leur taille, en fonction de l'abscisse dans l'échangeur.

[Distribution of drops : Variations of velocities of drops as a function of their radius, and as a function of the absciss.]

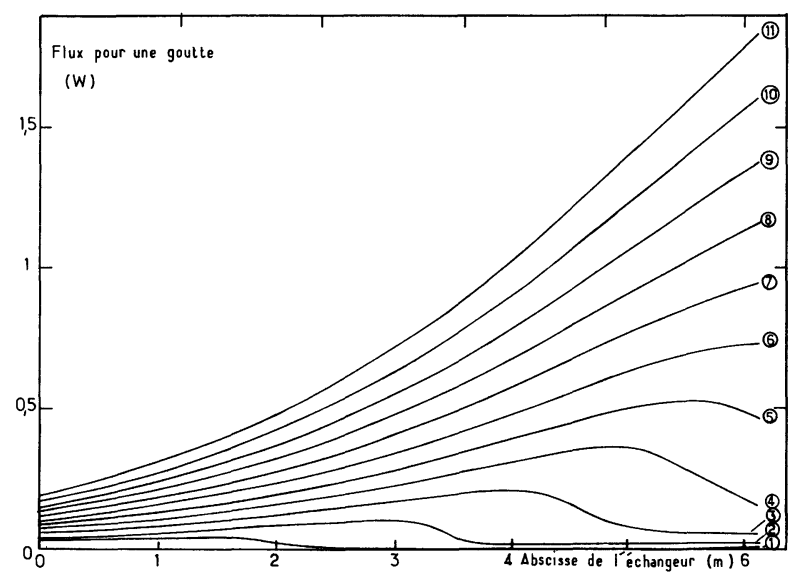

Fig. 8. - Cas d'une répartition de gouttes : Flux d'une goutte suivant sa taille, en fonction de l'abscisse dans l'échangeur.

[Distribution of drops : Heat transfer from drops as a function of absciss.]

est calculé de manière à ce que seule une quantité de chaleur équivalente à sa chaleur latente initialement disponible soit déstockée pour chaque goutte; cela implique, dans le cas d'une répartition de gouttes que, en bas de l'échangeur, les plus petites sont entièrement solidifiées tandis que les plus grosses comportent encore un noyau liquide.

4.3.2 Résultats et interprétation des courbes. - Le rayon optimum $R_{0}$ des gouttes est de $0,71 \mathrm{~mm}$, ce qui donne dans le second cas une plage comprise entre $1,06 \mathrm{~mm}$ et $0,355 \mathrm{~mm}$ de rayon. Le rayon de l'échangeur est de $2,23 \mathrm{~m}$, sa longueur est de $6,5 \mathrm{~m}$ ou de $6,12 \mathrm{~m}$ dans le premier et le deuxième cas respectivement. Une analyse similaire à celle qui a été faite en 4.2 pourrait être reprise, les différents résultats qualitatifs restant valables. Trois courbes sont présentées ici.

Les courbes 7 et 8 relatives à une répartition des tailles représentent respectivement les vitesses des gouttes et le flux qui en est extrait, en fonction de l'abscisse compté positivement depuis la section d'injection. Comme le montre la figure 7, les gouttes atteignent très rapidement leur vitesse d'équilibre ce qui est un élément défavorable, les vitesses relatives intergouttes augmentant le nombre de chocs, donc les coalescences éventuelles.

La courbe 8 se prête à la même interprétation que 3 , la seule particularité réside en ce que, pour une abscisse donnée, toutes les gouttes ne se trouvent pas dans le même état de solidification. On remarque en particulier que le flux extrait des plus petites devient rapidement négligeable.

La courbe 9 représente pour les deux options de ce calcul à savoir des gouttes dont $R_{1}=0,71 \mathrm{~mm}$ et des gouttes à répartition pseudo-gaussiennes entre 0,36

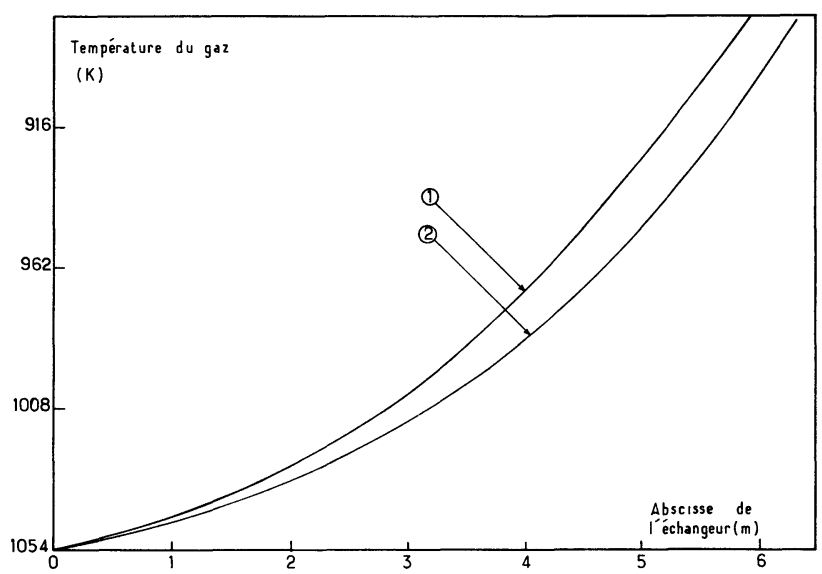

Fig. 9. - Température du gaz en fonction de l'abscisse dans l'échangeur. 1. - Répartition de gouttes. 2. - Gouttes monodimensionnelles.

[Gas temperature as a function of absciss : 1. - Distribution of drops. 2. - One drop size.]

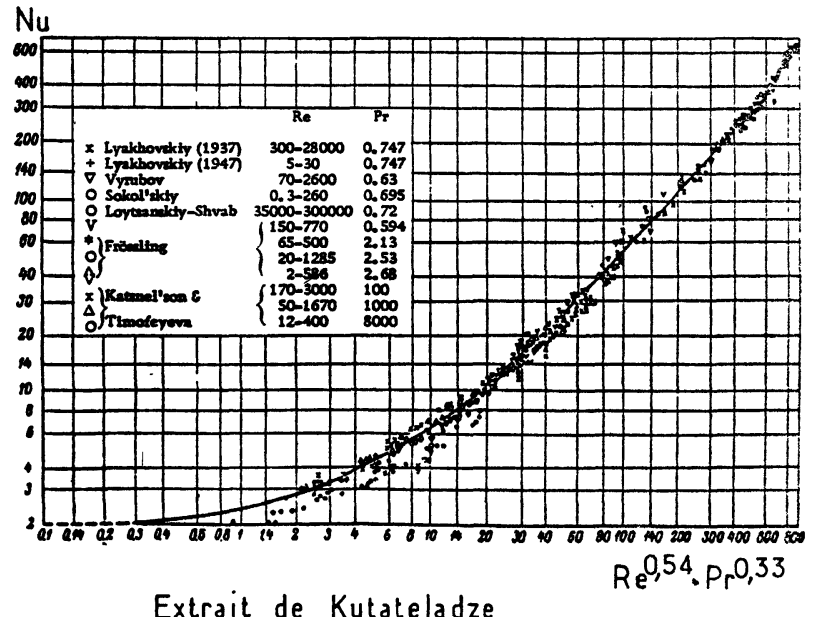

Fig. 10. - Données expérimentales sur le transfert convectif d'une sphère (extrait de Kutateladze).

[Experimental heat transfer data for a sphere (from Kutateladze).] 


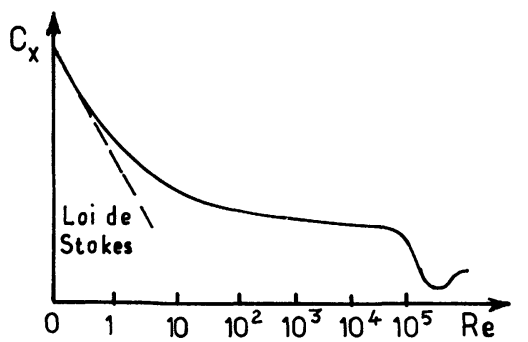

Fig. 11. - Coefficient de traînée en fonction du Reynolds.

[Drag coefficient as a function of Reynolds number.]

et $1,1 \mathrm{~mm}$, la température du gaz en fonction de l'abscisse dans l'échangeur. On trouve un meilleur échange dans le cas de tailles réparties, ce qui est normal car le calcul dans ce cas précis montre que le ralentissement des petites gouttes augmente la concentration volumique locale et donc le temps de séjour moyen de la masse injectée. A flux total égal dans les deux cas, la longueur de l'échangeur se trouve donc quelque peu réduite $(6,12$ au lieu de 6,50).

L'introduction d'une répartition des gouttes ne modifie donc pas fondamentalement les résultats obtenus pour les gouttes monodimensionnelles.

5. Conclusion. - L'échangeur direct gaz-gouttes présente un intérêt considérable par rapport aux échangeurs classiques à tubes; dans ces derniers, la formation, lors du déstockage, d'une croûte solide mauvaise conductrice altère d'autant plus fortement les performances de l'échangeur au cours du déstockage que le caloporteur de récupération est caractérisé par un coefficient convectif élevé. Or, dans un échangeur direct, le sel est éliminé au fur et à mesure de son utilisation, ce qui permet de maintenir constante la température de sortie du gaz aussi longtemps que le stock existe. D'autre part, la pulvérisation permet d'obtenir une surface d'échange quelconque qui compense un médiocre coefficient convectif par le gaz sans avoir à réaliser cette surface. Le prix de l'échangeur en est considérablement diminué.

Si on s'est limité ici à deux exemples adaptés à l'air à 20 bar, les programmes ont été faits pour n'importe quel type de gaz à n'importe quelle pression et température d'entrée.

Les résultats présentés ici font partie d'un programme de recherche concernant la pulvérisation des sels fondus, qui comporte la réalisation de dispositifs expérimentaux. Les premiers résultats permettront d'améliorer des modèles présentés ici. On doit aussi en retirer des enseignements sur les différents points en suspens : risques de coalescence, comportement vis-à-vis de la paroi, fluctuations de vitesse, déstabilisation de la valeur de la concentration constante dans une section droite donnée de l'échangeur, etc... Mais les premières expériences de pulvérisation où le sel est envoyé directement à l'air libre ambiant, ce qui provoque un choc thermique caractérisé par une brusque différence de température de l'ordre de $800^{\circ}$ montrent néanmoins le caractère bien monodimensionnel des gouttes. La constitution rapide d'une croûte écarte donc les possibilités de coalescence comme de subdivision.

Ces recherches ont été conduites en collaboration avec le laboratoire de Dynamique et Thermophysique des Fluides, associé au C.N.R.S. (L.A. 72) qui a mené à bien une étude sur les échangeurs directs à co-courant [13].

\section{Bibliographie}

[1] El'Kin, G. I. et Timufeyev, Yu B., Heat Transfer, Sov. Res. 8 (1976) $\mathrm{n}^{0}$ 5. Experimental study of the distribution of solid particles and of the local heat transfer between the gas and solids in a two-phase flow (with one particlesize).

[2] Gorbis, Z. R. et Kalender'Yan, Energiya Press, Moscow, 1975. Heat exchanges with disperse coolants.

[3] Chukhanov, Z. F., Int. J. Heat Mass Transfer 6 (1963) 691-701. 'Heat and mass transfer between gas and granular material.

[4] Chuknanov, Z. F., Int. J. Heat Mass Transfer 13 (1970) 18051817. Heat and mass transfer between gas and granular material.

[5] Chuckhanov, Z. F., Int. J. Heat Mass Transfer 14 (1971) 337-351, Heat and mass transfer between gas and granular material.

[6] Galloway, T. R. and Sage, B. H., Int. J. Heat Mass Transfer, 10 (1967) 1195-1210. Thermal and material transport from spheres.

[7] Savender, W. J. et Pei, D. C. T., Int. J. Heat Mass Transfer, 10 (1967) 529-539. The effect of fluid turbulence on the rate of heat transfer from spheres.

[8] Article à paraître dans la revue Générale de Thermique, suite à la réunion à la S.F.T. du 16 novembre 1978

[9] Chen, T. S. and Mucoglu, A., Int. J. Heat Mass Transfer, 21 (1977) 867-875. Analysis of mixed forced and free convection about a sphere.
[10] Kutateladze, S. S., Arnold, 1963. Fundamentals of heat transfer.

[11] Gaillard, J. L., thèse Doctorat $3^{\mathrm{e}}$ cycle, Paris VI. Conditions d'éclatement de gouttes en chute libre.

[12] Bonacina, C. et Comini, G., Int. J. Heat Mass Transfer, 16 (1973) 1825-1832. Numerical solution of phase-change problems.

[13] KAROUBY, N. et LARINI, M., à paraître. Etude d'une suspension de fines particules en cours de cristallisation.

[14] Pedroso, R. I., Domoto, G. A., J. Heat Transfer, U.S.A. (1973) 42-46. Perturbation solutions for spherical solidification of saturated liquids.

[15] Pedroso, R. I., Domoto, G. A., Int. J. Heat Mass Transfer, 16 (1973) 1037-1043. Inward spherical solidification. Solution by the method of strained coordinates.

[16] Ching Sun Huang, Yen Ping Shin, Chem. Eng. Sci, 30 (1975) 887-906. A perturbation method for spherical and cylindrical solidification.

[17] Riley, D. S., Smith, F. T., Poots, G., Int. J. Heat Mass Transfer, 17 (1974) 1507-1516. The inward solidification of spheres and circular cylinders. 